\title{
Vibration Modal Analysis of a Thin Folded Elastic Structure using a Levy-type Solution
}

\author{
Enrique Segovia and Belen Ferrer \\ Departamento de Ingeniería de la Construcción, Obras Públicas e Infraestructura Urbana, Universidad de Ali- \\ cante, Spain
}

Jaime Ramis

Departamento de Física, Ingeniería de Sistemas y Teoría de la Señal, Universidad de Alicante, Spain

Jose Martinez

Acústica Beyma, S.A. Polígono Industrial de Moncada, Calle Pont Sec, 1-C, Moncada, Valencia 46113, Spain

\author{
Jorge P. Arenas \\ Institute of Acoustics, Univ. Austral of Chile, Casilla 567, Valdivia, Chile
}

(Received 12 December 2011; Revised 21 June 2012; Accepted 2 August 2012)

In this technical note, an approximated analytical Levy-type solution for the system of differential equations that governs the vibration of a thin folded structure is presented. The folded structure is composed of thin plates and thin curved circular panels attached by their straight sides. Hinged and arbitrary boundary conditions at curved edges and straight edges, respectively, were used. In order to test the proposed analytical approach, numerical experiments using commercial finite element method (FEM) software were developed for a particular folded structure commonly found in Air Motion Transformer loudspeakers. It is found that the first two natural frequencies of this structure obtained with the analytical approach differed by less than $2 \%$ from those obtained using FEM. In addition, the analytical mode shape is almost identical to that obtained by FEM.

\section{INTRODUCTION}

Closed and open circular cylindrical shell structures have been widely used in civil, marine, aerospace, and petrochemical industry applications. ${ }^{1,2}$ These structures are frequently subjected to traverse loads, and the motion in this direction is considered the most significant one. In order to consider all possible boundary conditions, a combination of Navier's, ${ }^{3}$ Levy's, ${ }^{4}$ and superposition methods of solution was used in previous research. ${ }^{5}$ However, the superposition method gives a very complex solution.

In this paper, a simplified analytical solution for the equation of motion and for determining the natural frequencies is proposed. The approach uses a Levy-type solution based on classical thin shell theory ${ }^{6,7}$ for some particular boundary conditions. The approach is applied to a structure made of thin plates and curved cylindrical panels composed of layers of isotropic and homogeneous materials. Although the state-space technique has been commonly used to solve this kind of problem, a simple displacement function ${ }^{8}$ has been used in this work.

The state-space technique is a unified time-domain formulation that can be utilized for the analysis of many types of systems, including vibrating plates and shells. ${ }^{9-16}$ Systems of linear differential equations of motion can be modeled through state equations by identifying appropriate state variables. Further, the state-space method can be extended to the analysis of nonlinear systems. The method has also been widely used for active noise and vibration control in plates and shells. ${ }^{17}$

Specifically, a folded structure consisting of an arbitrary se-

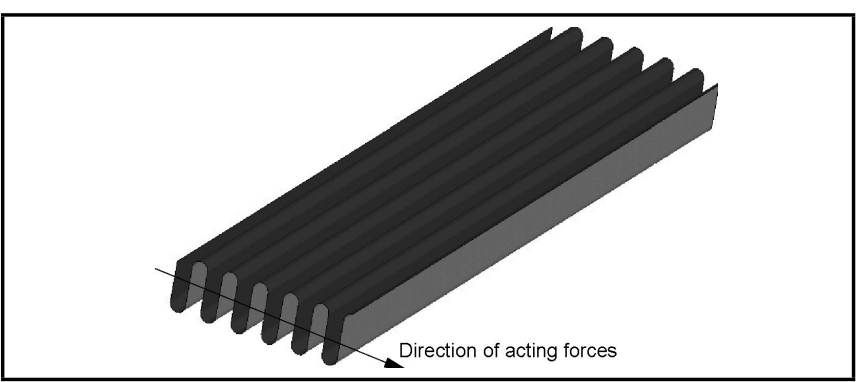

Figure 1. The folded structure and the direction of acting forces.

quence of thin plates and thin curved cylindrical panels connected by their straight edges and subject to traverse acting forces is considered here (see Fig. (1)). The final aim of this study is to obtain an approximate analytical solution for both the natural frequencies and displacement in the direction of forces. An example of the use of this type of structure is in the pleated moving assembly of an Air Motion Transformer (AMT) loudspeaker. ${ }^{18-20}$

The solution obtained in the next sections takes into account classic shell theory, assuming that thin sheets are considered and neglecting the shear strain. The differential equations of equilibrium for an open cylindrical shell ${ }^{11,21}$ of length $L$, radius $R$, and subjected to an arbitrary surface load $P\left(P_{x}, P_{y}, P_{z}\right)$, including the rotary inertia terms, are:

$$
\frac{\partial N_{x y}}{\partial y}+\frac{\partial N_{x}}{\partial x}=-P_{x}+I_{1} \ddot{u}-I_{2} \frac{\partial \ddot{w}}{\partial x}
$$

